\title{
Understanding the Structure and Mechanism of Formation of a New Magnetic Microbubble Formulation
} \author{
Eleanor Stride ${ }^{1 凶}$ \\ 1. Institute of Biomedical Engineering, Department of Engineering Science, University of Oxford, UK. \\ 2. Department of Bioengineering, Imperial College London, UK. \\ 3. Davy-Faraday Research Laboratory, The Royal Institution of Great Britain, London, UK. \\ 4. Imaging Sciences Department, Imperial College London, UK.
}

Joshua Owen' ${ }^{1}$ Bin Zhou ${ }^{2}$, Paul Rademeyer ${ }^{1}$, Meng-Xing Tang ${ }^{2}$, Quentin Pankhurst ${ }^{3}$, Robert Eckersley ${ }^{4}$ and

$\triangle$ Corresponding author: Email: eleanor.stride@eng.ox.ac.uk.

(C) Ivyspring International Publisher. This is an open-access article distributed under the terms of the Creative Commons License (http://creativecommons.org/ licenses/by-nc-nd/3.0/). Reproduction is permitted for personal, noncommercial use, provided that the article is in whole, unmodified, and properly cited.

Received: 2012.03.0I; Accepted: 2012.07.06; Published: 20I2.12.10

\begin{abstract}
Magnetic nanoparticles and ultrasound contrast agents have both been used as vehicles for therapeutic delivery. More recently, magnetic microbubbles have been developed as a new theranostic agent which combines the advantages of the individual carriers and overcomes many of their limitations. In a previous study of gene delivery using magnetic microbubbles, it was found that a combination of magnetic liquid droplets and non-magnetic phospholipid microbubbles produced higher transfection rates than magnetic microbubbles. The reasons for this were not fully understood, however. The aim of this study was to investigate the hypothesis that conjugation between the droplets and the microbubbles occurred. A combination of optical and fluorescence microscopy and ultrasound imaging studies in a flow phantom were performed. No interaction between magnetic droplets and microbubbles was observed under optical microscopy but the results from the fluorescence and acoustic imaging indicated that magnetic droplets and microbubbles do indeed combine to form a new magnetically and acoustically responsive particle. Theoretical calculations indicate that the driving force of the interaction is the relative surface energy and thus thermodynamic stability of the microbubbles and the droplets. The new particles were resistant to centrifugation, of comparable echogenicity to conventional ultrasound contrast agents and could be retained by a magnetic field (0.2T) in a flow phantom at centre line velocities of $\sim 6 \mathrm{~cm} \mathrm{~s}^{-1}$ and shear rates of $\sim 60 \mathrm{~s}^{-1}$.
\end{abstract}

Key words: Microbubbles, magnetic nanoparticles, therapeutic ultrasound

\section{Introduction}

\section{I.I. Background}

A large body of research in recent years has shown the considerable potential of both microbubbles and magnetic nanoparticles individually as effective theranostic agents (1-3). In addition to providing contrast enhancement in ultrasound imaging, coated microbubbles have been widely shown to improve cellular uptake of therapeutic material (4).
This effect, termed sonoporation, reversibly enhances the permeability of the surfaces of nearby cells and is thought to be produced by the oscillations of microbubbles under ultrasound excitation (5). Close proximity between the microbubbles and target cells is required for therapeutic effects to be realised without unwanted damage and at moderate ultrasound intensities (6). Similarly, adequate numbers of microbubbles are required at the target site, but in- 
creasing microbubble concentration can pose an increased risk of embolism and shield target tissue from ultrasound exposure (7). Efficient bio-chemical targeting of microbubbles still represents a considerable challenge in vivo and surface architectures that maximise ligand exposure also boost presentation of immunogenic compounds that leads to early particle clearance or a hypersensitivity response $(2,7)$.

Magnetic particles, on the micro and nano scale, have been investigated for the delivery of therapeutic agents for decades (8-10). Magnetic nanoparticles have also been used more recently for gene delivery. The association of genes with magnetic nanoparticles for magnetically guided nucleic acid delivery has been termed "magnetofection" (11). Magnetic nanoparticles can be used for targeting as they can be externally controlled by a magnetic field and can also be visualised by Magnetic Resonance Imaging (MRI). However, the small size of the magnetic nanoparticles means they cannot be retained as individual particles under physiologically relevant flow conditions (12). A combination of these delivery methods in the form of magnetic microbubbles has the potential to overcome many of these limitations. Magnetic microbubbles can be visualised using ultrasound imaging, localised using an externally applied magnetic field and thus exploit the combined effects of magnetofection and sonoporation for therapeutic delivery.

In 2000, Soetanto et al. electrostatically attached magnetic microparticles, coated with charged stearates, via a calcium ion to microbubbles also formed with charged stearates $(13,14)$. According to the literature however, the large size of the microparticles and the effect conditions in vivo would have upon the electrostatic interactions were causes for concern. Magnetic microbubble formulations have since been developed for dual purpose ultrasound and MRI contrast agents and as drug delivery vehicles (15-18). In the case of the latter, Stride et al. (19) published a study in which magnetic microbubbles were used for gene delivery to Chinese hamster ovary cells. Different formulations of magnetic microbubbles, non-magnetic microbubbles and magnetic liquid droplets were co-injected with naked plasmid DNA encoding for luciferase and the cells exposed to a magnetic field, ultrasound or both. In addition, the experiments were performed with the cells on either the upper or lower surface of the culture plate so that in the case of the former, buoyant bubbles would be in contact with the cells. In the case of the latter, however, they would be separated by a distance of $2 \mathrm{~mm}$ unless they were magnetically-responsive in which case they would be translated downwards in the presence of a magnet. It was found that the highest rates of transfection were achieved with simultaneous exposure to ultrasound and a magnetic field, with the cells on the lower surface and with 2 formulations (Figure 1): magnetic microbubbles (consisting of a gas core coated in an oil suspension of magnetic nanoparticles and stabilised by a phospholipid coating) and secondly non-magnetic microbubbles mixed with magnetic droplets (droplets of ferrofluid, stabilised by a phospholipid coating). This combination of magnetic droplets and non-magnetic microbubbles produced a higher degree of transfection than magnetic microbubbles (19).

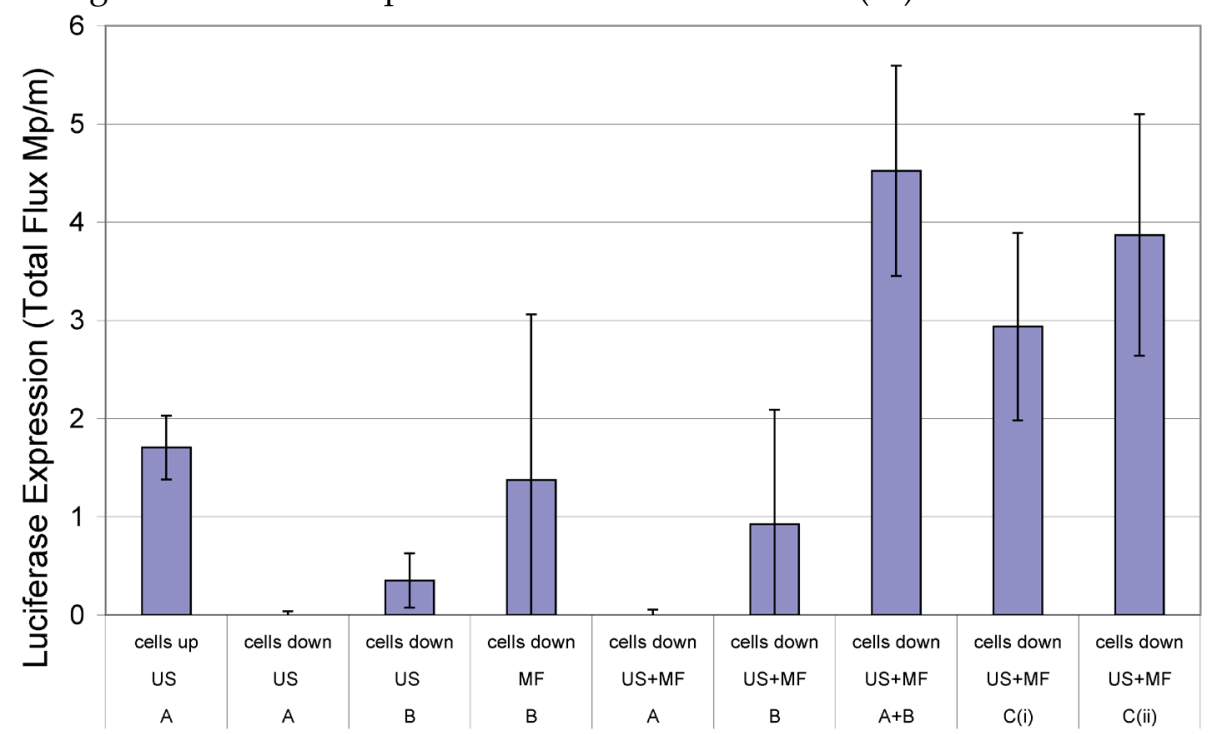

Figure I: Transfection enhancement as determined from Luciferase expression by Chinese hamster ovary cells $24 \mathrm{~h}$ after exposure to naked plasmid DNA with: an applied magnetic field (MF) and/or ultrasound (US) (IO s of pulsed insonation at I MHz, I MPa peak-to-peak, $10 \mathrm{kHz}$ PRF) in the presence of non-magnetic bubbles (A) and/or magnetic liquid droplets (B) or magnetic microbubbles (Ci or $\mathrm{Cii}$ ) with cells located on either the upper or lower surface of the cell culture chamber. Full experimental details may be found in [19]. Reproduced with permission from Elsevier. 


\section{I.2. Motivation and Objectives}

The reason why magnetic droplets mixed with microbubbles produced transfection was not fully understood, since it was expected that the droplet/microbubble mixture would produce similar results to droplets alone. Two hypotheses were proposed: first that magnetic droplets were somehow coupled to microbubbles, although this was not evident under microscopic examination; or second that magnetic droplets had a sensitising effect upon the cells which amplified the effect of the microbubbles, overcoming the fact that they were not in close proximity to the cells (19). The aim of this study was to investigate these observations further and in particular to test the first hypothesis through examination of microbubble/droplet mixtures under optical and fluorescence microscopy and to examine their response to a combination of magnetic and ultrasound fields in a flow phantom to determine their ability to be imaged and retained under physiologically relevant conditions.

\section{Materials and Methods}

L-a-phosphatidylcholine and petroleum ether, pure (boiling range $180-280^{\circ} \mathrm{C}$ ) were purchased from Sigma Aldrich, Poole, UK. The ferrofluid suspension of $10 \mathrm{~nm}$ spherical magnetite nanoparticles in isoparaffin (10\% volume fraction) was purchased from Liquids Research, Bangor, UK. The fluorophore BODIPY $\mathrm{C}_{10}$ was synthesised and kindly provided by the Chemistry Department, Imperial College London (20).

\section{I. Bubble preparation}

Non-magnetic microbubbles were prepared with L-a-phosphatidylcholine based upon the technique of Stride et al. [19]. $15 \mathrm{mg}$ of the lipid powder was weighed into a vial rinsed with surgical spirit (BP Unichem, Surrey, UK). Deionised water $(15 \mathrm{ml})$ was then added to the vial and the mixture sonicated for $30 \mathrm{~s}$ with an ultrasonic cell disruptor (XL 2000, probe diameter 3 mm, Misonix Inc. Farmingdale, NY, USA). This solution was then vigorously shaken for an additional $30 \mathrm{~s}$. Magnetic microbubbles were prepared via the same method but $15 \mu \mathrm{l}$ of the magnetic nanoparticle suspension was added to the lipid mixture after sonication. This was followed by an additional $30 \mathrm{~s}$ of sonication and $30 \mathrm{~s}$ of manual shaking. Magnetic droplets were also prepared by a similar method but omitting manual shaking and during sonication care was taken to keep the probe tip well below the liquid surface to avoid entraining gas.

Fluorescent formulations, magnetic and non-magnetic, were prepared via the following method. L-a-phosphatidylcholine dissolved in chloroform $(80 \mu \mathrm{l})$ and BODIPY $\mathrm{C}_{10}$ dissolved in chloroform $(2 \mu \mathrm{l})$ were added to a glass vial and agitated using a vortex mixer for 30s (VWR digital vortex mixer running at $1500 \mathrm{rpm}$ ). This mixture was then dried with a constant nitrogen flow for $30 \mathrm{~min}$. Deionised water $(2 \mathrm{ml})$ was added followed by bath sonication (Crest Ultrasonics, Model No 950HTAG, Trenton, NJ, USA) at room temperature for $1 \mathrm{~min}$. The solution was transferred to a plastic vial followed by isoparaffin $(2 \mu \mathrm{l})$, with or without magnetic nanoparticles, and the solution was probe sonicated and then shaken manually, each for 30s.

The phospholipid microbubbles were mixed with the droplets (magnetic and non-magnetic) as follows: magnetic droplets $(0.5 \mathrm{ml})$ were extracted with a $1 \mathrm{ml}$ syringe followed by microbubbles $(0.5 \mathrm{ml})$ and the mixture was inverted 3 times to allow the bubbles to pass through the magnetic droplet mixture. Three batches of each type of particle suspension were prepared for the experiments.

\subsection{Optical Microscopy}

The microbubbles, droplets and microbubble-droplet mixture were examined using optical microscopy to determine the particle size distribution and concentration. $10 \mu \mathrm{l}$ was removed from the sample solution and examined on a haemocytometer (Bright-Line, Hausser Scientific, Horsham, PA, USA). Images were obtained at 400x magnification with a standard light microscope (Bresser Optical Microscope No 0510119). Samples were taken from each of the 3 batches and 30 images were taken from each sample. The bubble size distribution and concentration were then obtained using purpose written image analysis software in MatLab (2010B, The MathWorks, Natick, MA, USA) (21). All experiments were performed at the ambient temperature in accordance with the conditions under which bubbles were prepared in [19].

\subsection{Fluorescence experiments}

Fluorescence microscopy was used to investigate the interaction between magnetic droplets and microbubbles. Images were obtained with an excitation wavelength of $490 \mathrm{~nm}$ and an absorption wavelength of $525 \mathrm{~nm}$ (Leica DMI6000B, FITC filter set). The manufacturer supplied software (Leica AF7000) was used to analyse the images. To analyse the non-magnetic droplets, images were acquired with a Nikon Eclipse Ti microscope (Nikon Instruments Europe B.V.) using a FITC filter set at the same wavelengths as above. In each case observations were made from two separate samples of microbubbles and 
fluorescent droplets prepared independently.

\subsection{Capillary flow model}

A $200 \mu \mathrm{m}$ diameter cellulose capillary tube $(\mathrm{Cu}-$ prophan RC55 8/200, Membrana GMBH) was suspended in a water bath $\left(23^{\circ} \mathrm{C}\right)$ and perfused with magnetic droplets and microbubbles, via a syringe into the needle of which the capillary had been inserted. Since the tube was both acoustically and optically transparent, the flowing particles were observed using an optical microscope with a water immersion objective (LUMPLFLN 40XW Olympus Key Med Southend, Essex UK; giving 400x magnification when mounted on a World Precision Instruments microscope, H602-240, Sarasota, Fl, USA with a 10x eye piece). An N52 grade NdFeB permanent magnet (NeoTexx, Berlin, Germany $10 \mathrm{~mm} \times 10 \mathrm{~mm} \times 25 \mathrm{~mm}$ ) with a transverse magnetisation of $0.75 \mathrm{~T}$ was held approximately $1 \mathrm{~mm}$ away from the capillary tube wall. Flow rates up to $1 \mathrm{~mm} / \mathrm{s}$ could be applied. A video was recorded of the mixtures flowing in the tube using a camera (ThorLabs USB 2.0 digital camera). Multiple perfusions were performed with samples from different batches of microbubbles and magnetic droplets.

\subsection{Ultrasound Flow Phantom}

In addition to further demonstrating the interaction between bubbles and droplets, it was important to investigate their echogenicity and also their ability to be magnetically retained under flow. The apparatus was set up as shown in Figure 2. A latex tube $(8 \mathrm{~mm}$ inner diameter with a wall thickness of $2 \mathrm{~mm}$ ) was suspended in a water bath and connected to a programmable syringe pump (Al-1000, World Precision Instruments, Herts. UK) at one end and an open collection vessel at the other. A section of the tube was positioned so that it was parallel to the base of the bath with a gap of approximately $3 \mathrm{~cm}$ to allow for the insertion of a magnet. This took the form of a Halbach array consisting of $5 \mathrm{~N} 52$ grade $\mathrm{NdFeB}$ permanent magnets $(10 \mathrm{~mm} \times 10 \mathrm{~mm} \times 25 \mathrm{~mm}$, supplied by NeoTexx, Berlin, Germany) with transversal magnetisations $(0.75 \mathrm{~T})$ at angles of $90^{\circ}$ from one to the next in an aluminium frame. The bubble formulations were imaged using an Ultrasonix RP500 ultrasound system using an array transducer with a centre frequency of $2.5 \mathrm{MHz}$ (PA4-2/20). This was positioned above the section of tube under which the Halbach array was located. The flow rate through the tube was varied using the syringe pump and the local velocity profile in the imaging plane was determined via particle image velocimetry. As soon as an acoustic signal was observed from the flowing microbubbles, the Halbach array was inserted. Experiments were performed for non-magnetic microbubbles, magnetic microbubbles, magnetic droplets and finally magnetic droplets mixed with microbubbles. Two solutions were prepared independently for each flow experiment and 5 perfusions performed with each.

\subsection{Flow estimation}

In the experiments, measurements of the axial velocity profile in the imaged region of the flow phantom were obtained via an ultrasonic particle image velocimetry (Echo PIV) method. Echo PIV is a non-invasive technique for flow characterisation, which overcomes the need for optical access. A series of consecutive ultrasound images are recorded. The images are then subdivided in smaller domains or interrogation areas and the correlation between two interrogation areas of consecutive frames is calculated. An advantage of this technique is its ability to measure the whole flow field simultaneously. Image pairing is performed through a spatial cross-correlation $R(s)$ of the interrogation windows $W_{1}(x) I_{1}(x)$ and $W_{2}(x) I_{2}(x)$ as given in equation 1 (22).

$$
R(s)=\int W_{1}(x) I_{1}(x) W_{2}(x+s) I_{2}(x+s) d x
$$

Where $W_{1}$ and $W_{2}$ are functions that determine the interrogation windows, and $I_{1}$ and $I_{2}$ are the images. Velocity magnitude is denoted by colour, as shown in Figure 3. The volume flow rate is estimated from the measured asymmetric axial velocity profiles by means of the $\cos \theta$ method (23). For the $\cos \theta$ method, the flow profile is split into two equal parts at the centreline, $V_{1}(r)$ and $V_{2}(r)$. Each part of the profile was integrated over half of the surface of the tube. The volume flow rate is then approximated from the sum of both contributions:

$$
Q=\pi \int_{0}^{R} V_{1}(r) r d r+\pi \int_{0}^{R} V_{2}(r) r d r
$$

Where $R$ is the inner radius of the tube.

In order to estimate the shear rate at the wall in the region above the magnetic array a Poiseuille flow profile was assumed and thus:

$$
\gamma=\frac{8 V}{2 r}
$$

Where $\gamma$ is the shear rate measured in $\mathrm{s}^{-1}, V$ is the centreline flow velocity obtained from PIV and $r$ is the radius of the tube. 


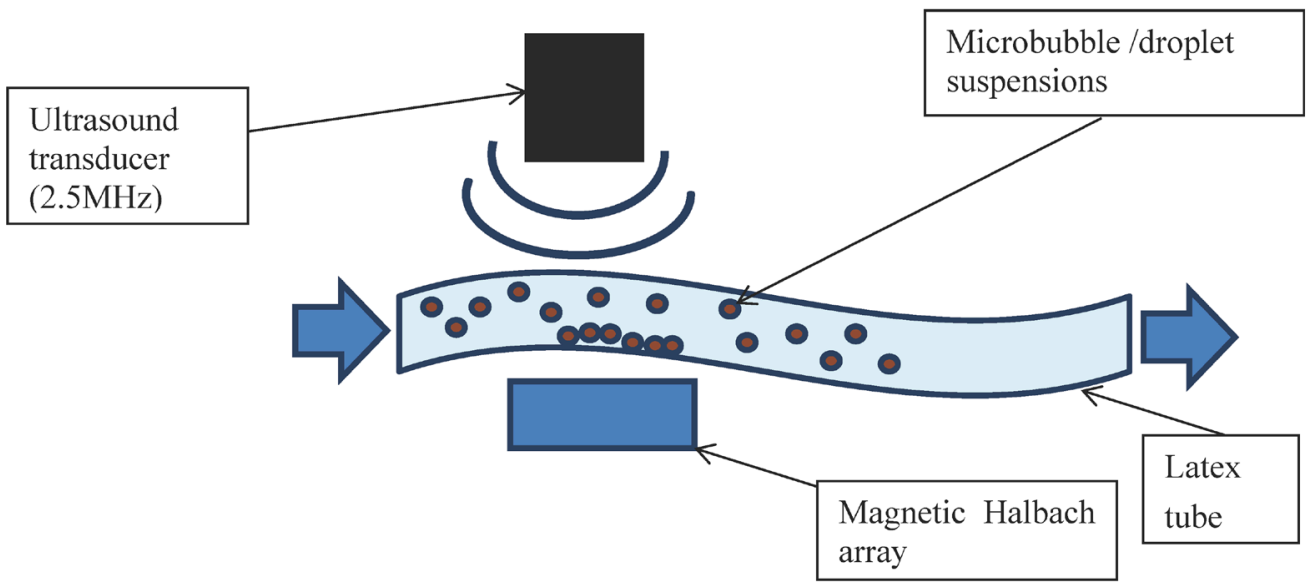

Figure 2: Schematic of the ultrasound flow phantom: a latex tube immersed in a water bath with a variable internal flow rate controlled by a syringe pump. Microbubble/droplet mixtures were injected into the tube upstream and images obtained using an ultrasound imaging probe. A magnetic Halbach array was inserted $2 \mathrm{~cm}$ below the latex tube to investigate retention of the microbubbles against the flow.

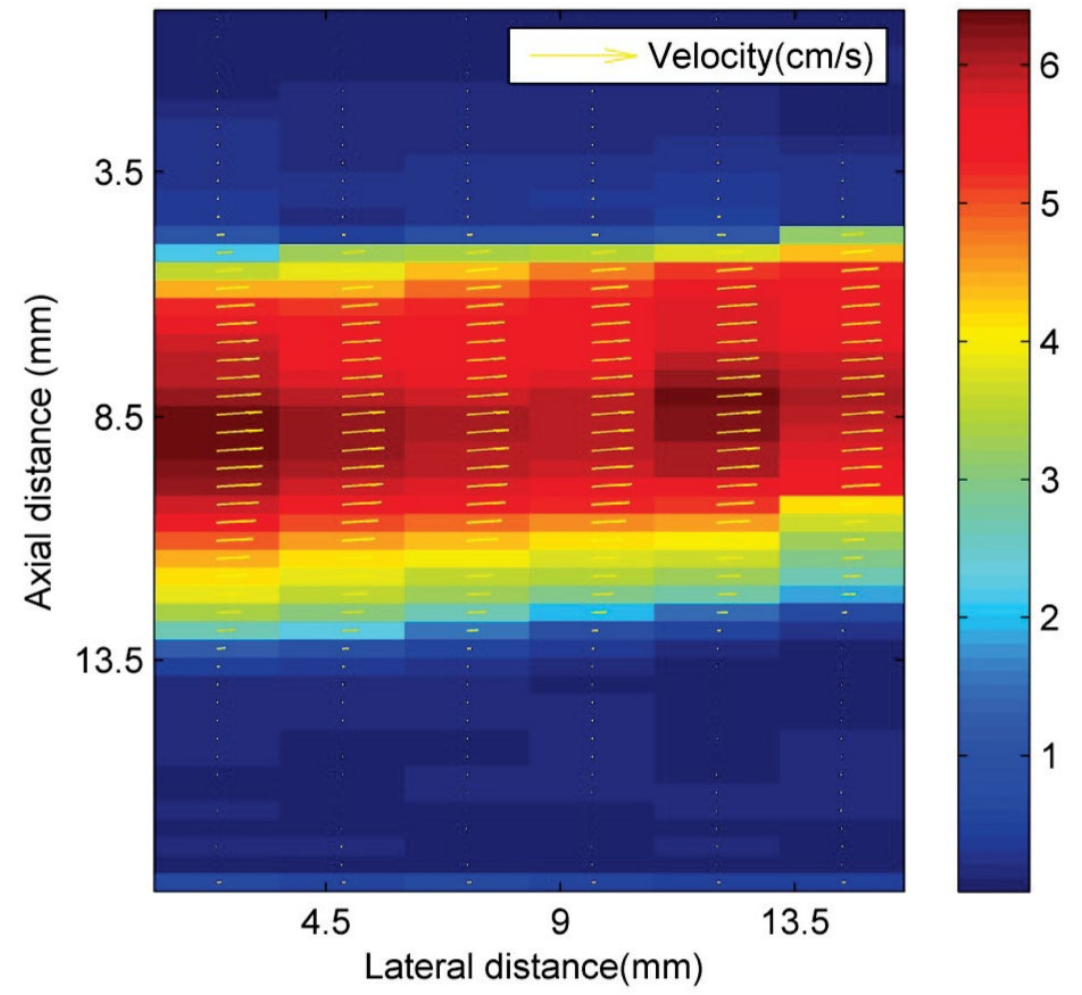

Figure 3: Ultrasonic particle image velocimetry (Echo PIV) velocity profiles $\left(\mathrm{cm} \mathrm{s}^{-1}\right)$ in the area above the magnetic array in the latex tube flow phantom.

\section{Results}

\section{I. Optical Microscopy}

L-a-phosphatidylcholine (non-magnetic) microbubbles were prepared as described above and examined on a haemocytometer. Microbubbles could be discerned at the top of the slide (Figure $4 \mathrm{~A}$ ). The bubbles had a range of sizes $(0.6-9.6 \mu \mathrm{m})$ and an av- erage concentration of $9.64 \times 10^{7}$ microbubbles $/ \mathrm{ml}$ (Figure $4 \mathrm{~F}$ ).

Optical microscope images showed the magnetic droplet solution as a mixture of particles of differing size (Figure $4 \mathrm{~B}$ ), which responded by forming chains and moving upon application of a magnetic field, as shown in Figure $4 \mathrm{C}$. The magnetic droplets were observed at the bottom of the slide and floating within the solution but none were observed at the top of the 
slide. Non-magnetic droplets showed similar structures but many of these rose to the top of the slide or floated in solution (Figure $5 \mathrm{~A}$ ). This indicated that the magnetic nanoparticles caused a decrease in the buoyancy of the droplets as might be expected given the relative densities of isoparaffin and magnetite. There was also a difference in colour due to the presence of magnetic nanoparticles.

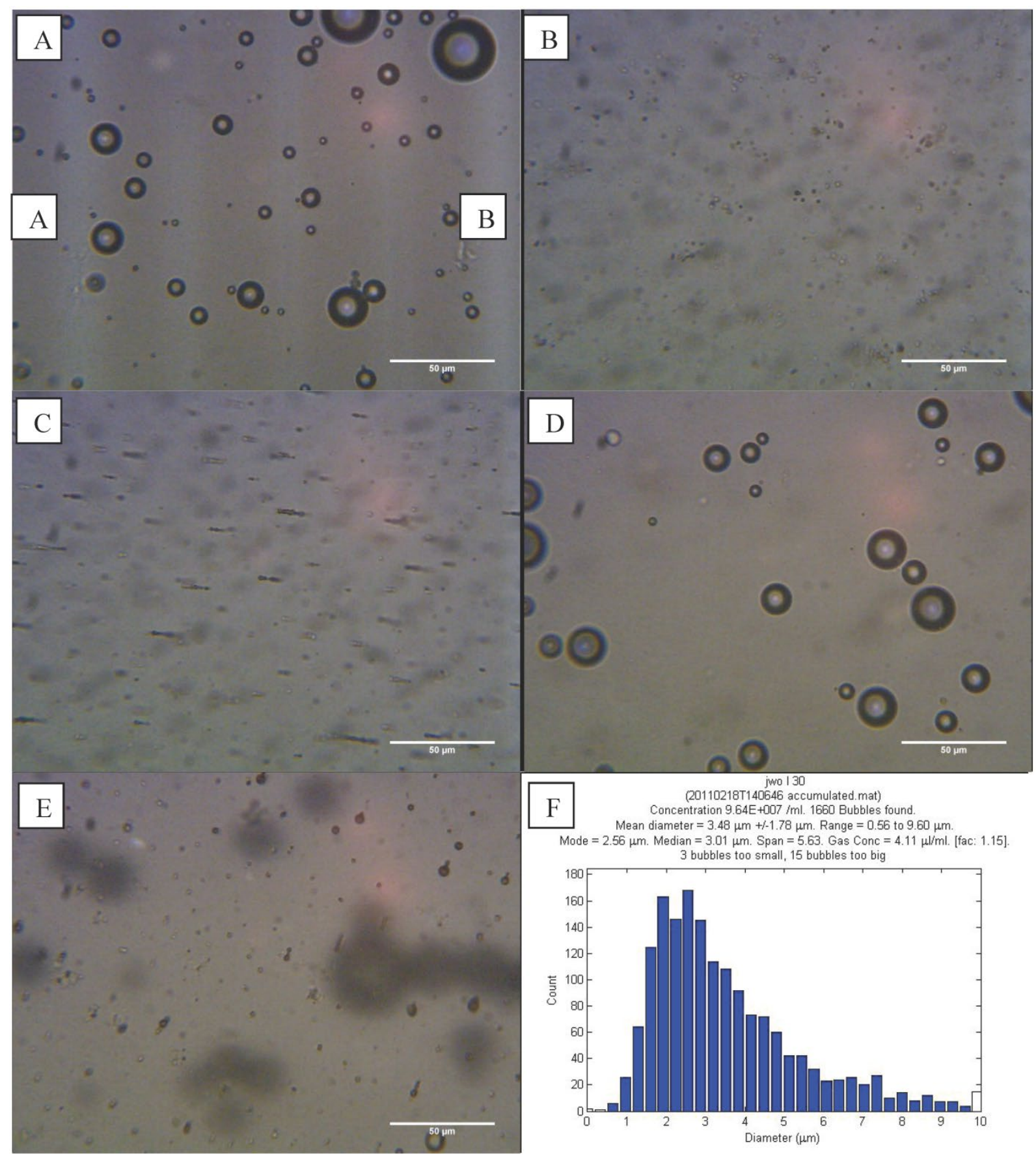

Figure 4: Optical images at 400x magnification of A) phospholipid microbubbles and B) magnetic droplets before and C) after the application of a magnetic field showing magnetic droplets in the micrometre scale responding to a magnetic field. D) and E) Phospholipid microbubbles combined with magnetic droplets showing $D$ ) the top of the slide with large buoyant microbubbles that did not respond to a magnetic field and E) particles below the large microbubbles (shown out of focus) lining up upon the application of a magnetic field. Scale bar in all images is $50 \mu \mathrm{m}$. F) Example of size distribution plot (in this case for non-magnetic microbubbles) obtained from the image processing software. 


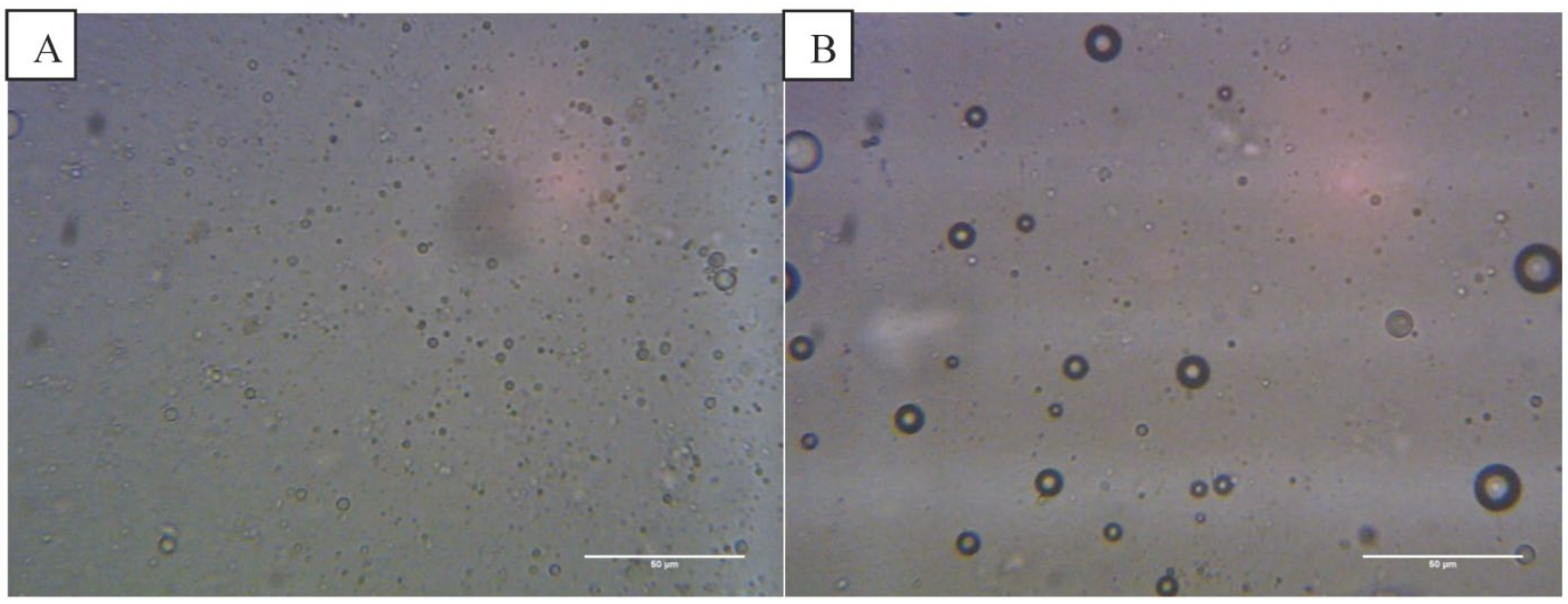

Figure 5: Optical microscope images (400x magnification) of: A) Non-magnetic droplets imaged at the top of the haemocytometer and B) non-magnetic droplets mixed with microbubbles in the same focal plane. Two separate populations of microbubbles and droplets on the micrometre scale can be observed. Scale bar in all images is $50 \mu \mathrm{m}$.

The magnetic droplet/microbubble mixture was placed directly onto the haemocytometer. Some relatively large microbubbles could be seen at the top of the slide which did not respond to a magnetic field (Figure $4 \mathrm{D}$ ). Throughout the solution however there were spherical particles which were magnetically responsive. The structure seemed to be similar to the solution of magnetic droplets and no interaction between the magnetic droplets and the microbubbles could be discerned. Figure $4 \mathrm{E}$ shows the magnetic droplets responding to a magnetic field with microbubbles out of focus above the droplets. Similarly, no interaction could be discerned between non-magnetic droplets and microbubbles as shown in Figure 5 B.

\subsection{Fluorescence Microscopy}

Fluorescent magnetic droplets were examined in water by fluorescence microscopy. A mixture of vesicles of differing size were observed by phase contrast (Figure $6 \mathrm{~A}$ ) and seen to fluoresce upon use of the FITC filter set (Figure 6 B). Non-magnetic phospholipid microbubbles were examined to confirm that no fluorescence was observed. Upon mixing the microbubbles with the fluorescent magnetic droplets, however the microbubbles were now observed to fluoresce as shown in the phase contrast (Figure $6 \mathrm{C}$ ) and fluorescence (Figure $6 \mathrm{D}$ ) images below. The microbubbles continued to fluoresce after centrifugation $(10,000 \mathrm{RPM}$ for $15 \mathrm{~min})$ indicating a strong interaction between the microbubbles and the droplets. This finding was confirmed with 3 separate samples.

Fluorescent isoparaffin droplets, without magnetic nanoparticles, were also mixed with phospholipid microbubbles. The microbubbles were observed by phase contrast, as shown in Figure $7 \mathrm{~A}$, and the microbubbles are also seen to fluoresce as shown in Figure $7 \mathrm{~B}$. The results are similar to those shown in Figure 6 with fluorescent magnetic droplets indicating that the magnetic nanoparticles were not essential to the interaction. Again this finding was confirmed with 3 independently prepared samples.

\subsection{Capillary flow}

The magnetic droplet/microbubble mixture was then injected into the optical flow phantom in order to observe whether the particles could be retained by a magnetic field (Figure $8 \mathrm{~A}$ ). Upon insertion of the magnet into the water bath the majority of objects were observed forming chains and translating towards the magnet (Figure $8 \mathrm{~B}$ ). Retention of microbubbles was observed at centre line velocities up to $1 \mathrm{~mm} / \mathrm{s}$, which was the maximum velocity of the system corresponding to a shear rate of $20 \mathrm{~s}^{-1}$. This observation was consistent in all samples tested (minimum of at least 3).

\subsection{Ultrasound flow phantom}

Ultrasound imaging in a larger scale flow phantom was carried out in order to examine the echogenicity of the particles and whether or not retention was possible in a larger vessel at higher flow rates. First, L-a-phosphatidylcholine magnetic microbubbles were injected. As soon as the magnetic microbubbles were observed on the ultrasound scanner (Figure 9 A), the Halbach array was inserted and retention of magnetic microbubbles was observed as shown in Figure $9 \mathrm{~B}$ and C. Upon removal of the Halbach array, the magnetic microbubbles were removed by flow in the tube (Figure $9 \mathrm{D}$ ). Retention of 
magnetic microbubbles was observed for flow rates of up to $\sim 6 \mathrm{~cm} / \mathrm{s}$ (Figure 3). This corresponds to shear rates of between 20 and $60 \mathrm{~s}^{-1}$. For each flow rate, retention was confirmed with a minimum of 3 injections of magnetic bubbles.

L-a-phosphatidylcholine microbubbles alone were injected into the flow phantom and could be easily observed on the scanner (Figure $10 \mathrm{~A}$ ) but, as would be expected, no retention was observed upon insertion of the Halbach array. Magnetic droplets were injected into the flow phantom and, also as expected, no acoustic signal was detected (Figure $10 \mathrm{~B}$ )
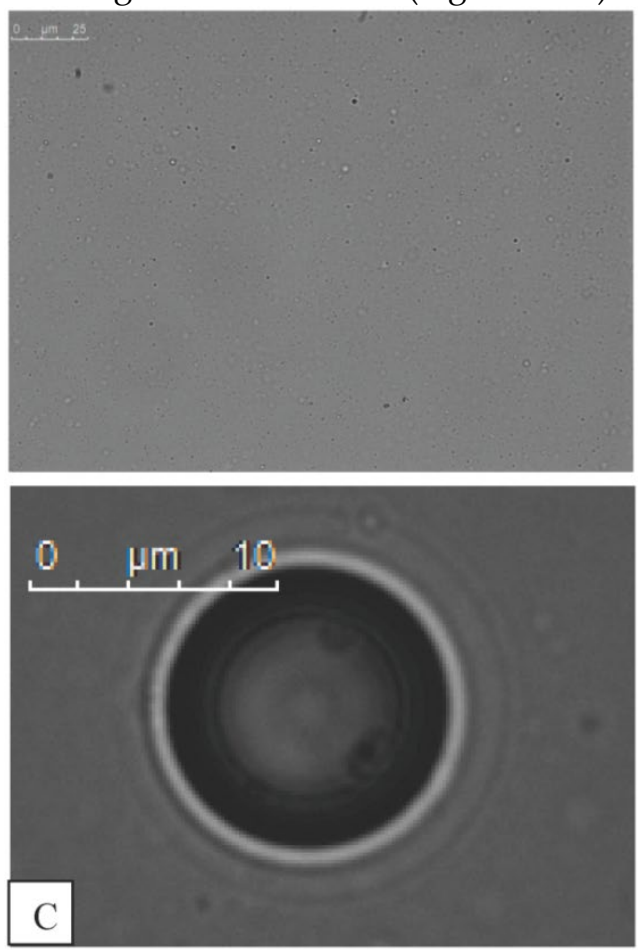

and consequently again upon insertion of the Halbach array no effect was observed. Finally, the magnetic droplet/microbubble mixture was injected into the flow phantom. A clear signal from particles in the tube could be seen on the scanner as shown in Figure 10 and upon insertion of the Halbach array retention was observed at flow rates up $\sim 6 \mathrm{~cm} / \mathrm{s}$ as shown in Figure $10 \mathrm{D}$. This was observed consistently for three injections from two different mixtures. No fluorescent formulations were used in the flow phantom experiments.
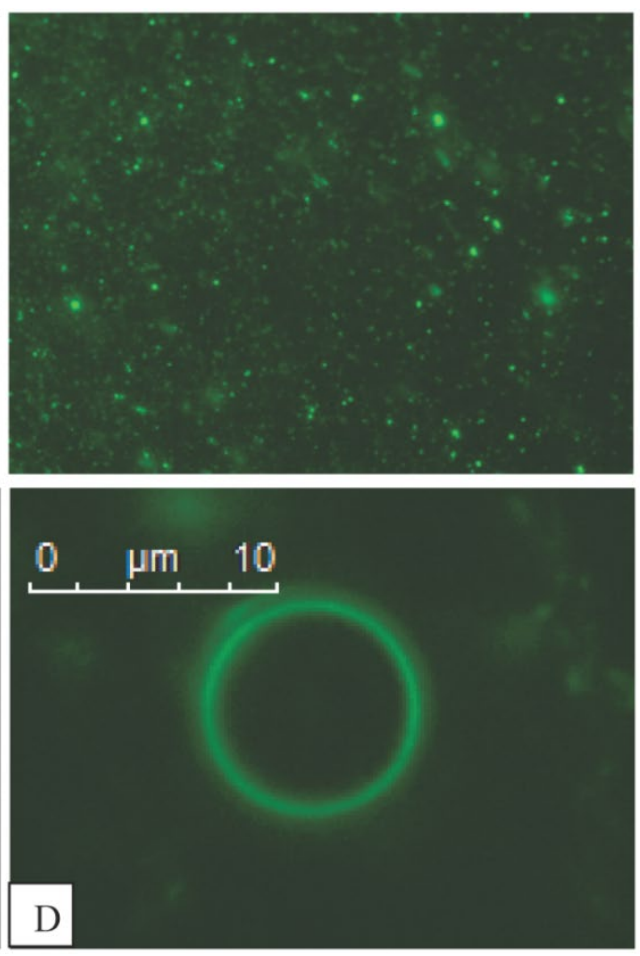

Figure 6: Fluorescent magnetic droplet mixture under optical microscopy shown in phase contrast in A) and fluorescence image in B) at 100x magnification (scale bar in $A$ is $25 \mu \mathrm{m}$ for images $A$ and B). C) Phase contrast image of a microbubble (400x magnification) in a mixture of fluorescent magnetic droplets and D) a fluorescence image of the same microbubble now fluorescing indicating the microbubble is interacting with fluorescent magnetic droplets.

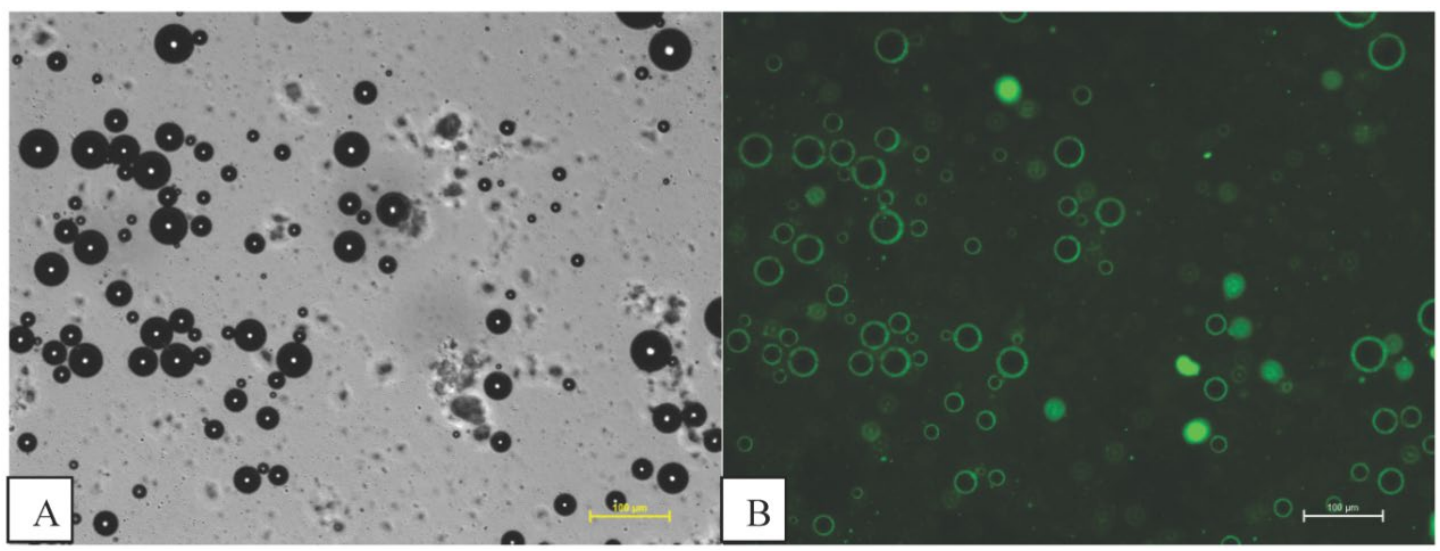

Figure 7: Microbubbles mixed with fluorescent isoparaffin droplets fluorescing indicating that the microbubbles are interacting with the oil based as well as the magnetic droplets. Scale bars are shown in the diagram $(100 \mu \mathrm{m})$. 


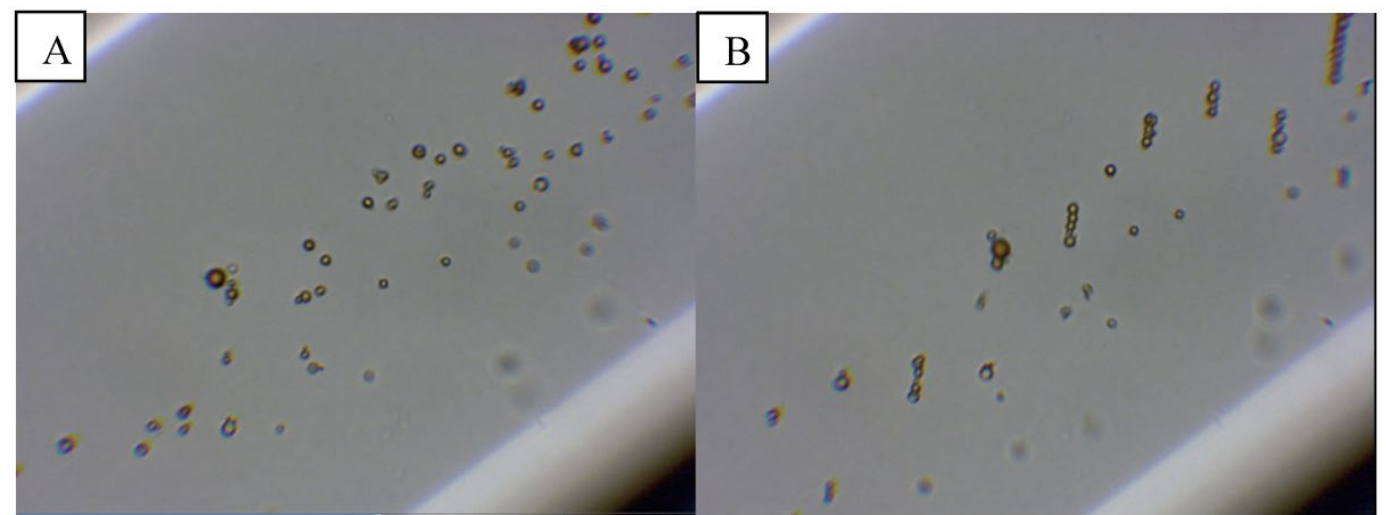

Figure 8: Microbubbles mixed with magnetic droplets in a $200 \mu \mathrm{m}$ capillary tube before A) and B) after the application of a magnetic field.

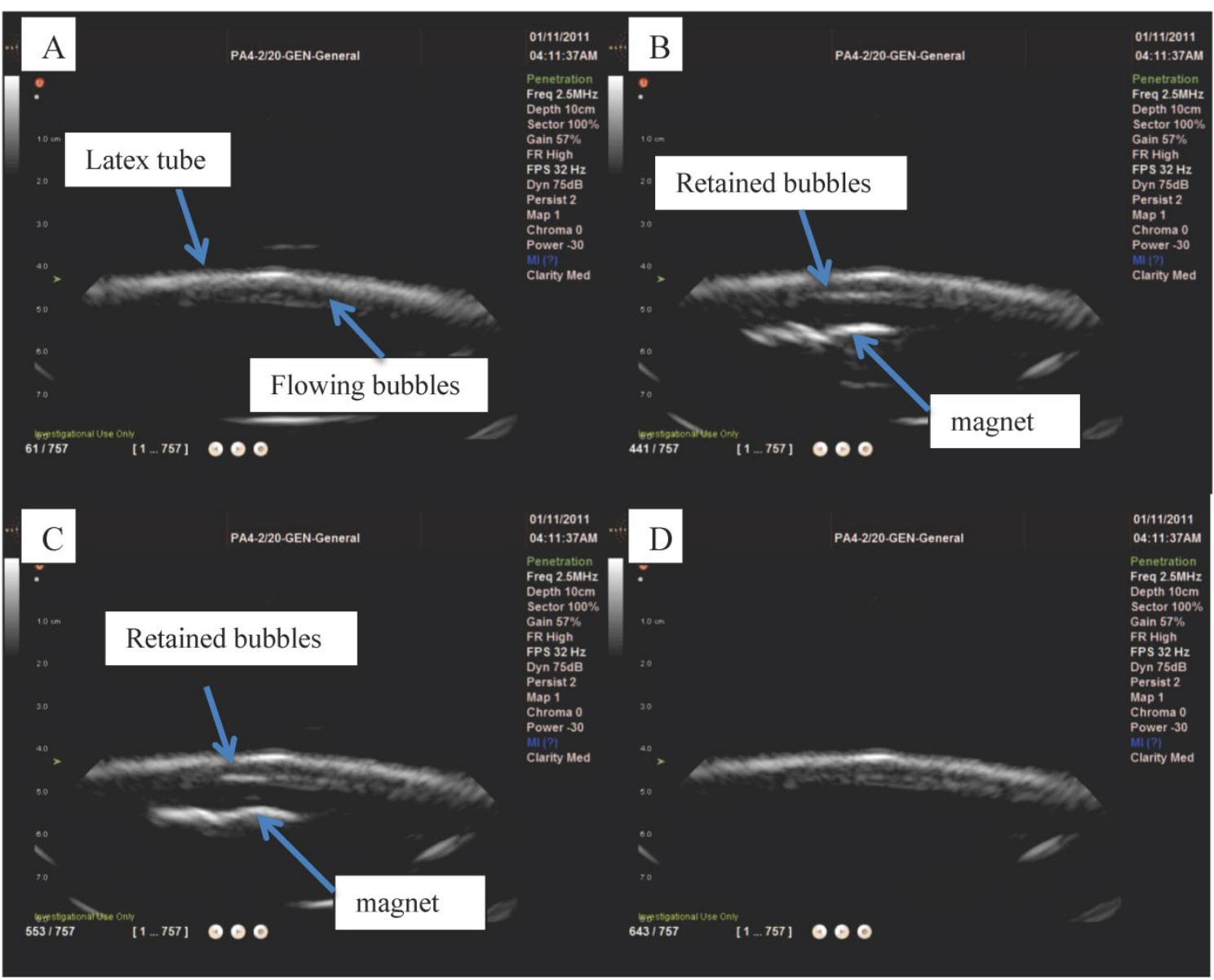

Figure 9: Ultrasound images from the flow phantom study: A) magnetic microbubbles flowing through an 8mm inner diameter latex tube, B) a magnetic array is brought underneath and magnetic microbubbles are retained at the lower surface of the tube and C) the number of bubbles retained increases over time. When the magnetic array is removed (D) the microbubbles are removed by the flow. 

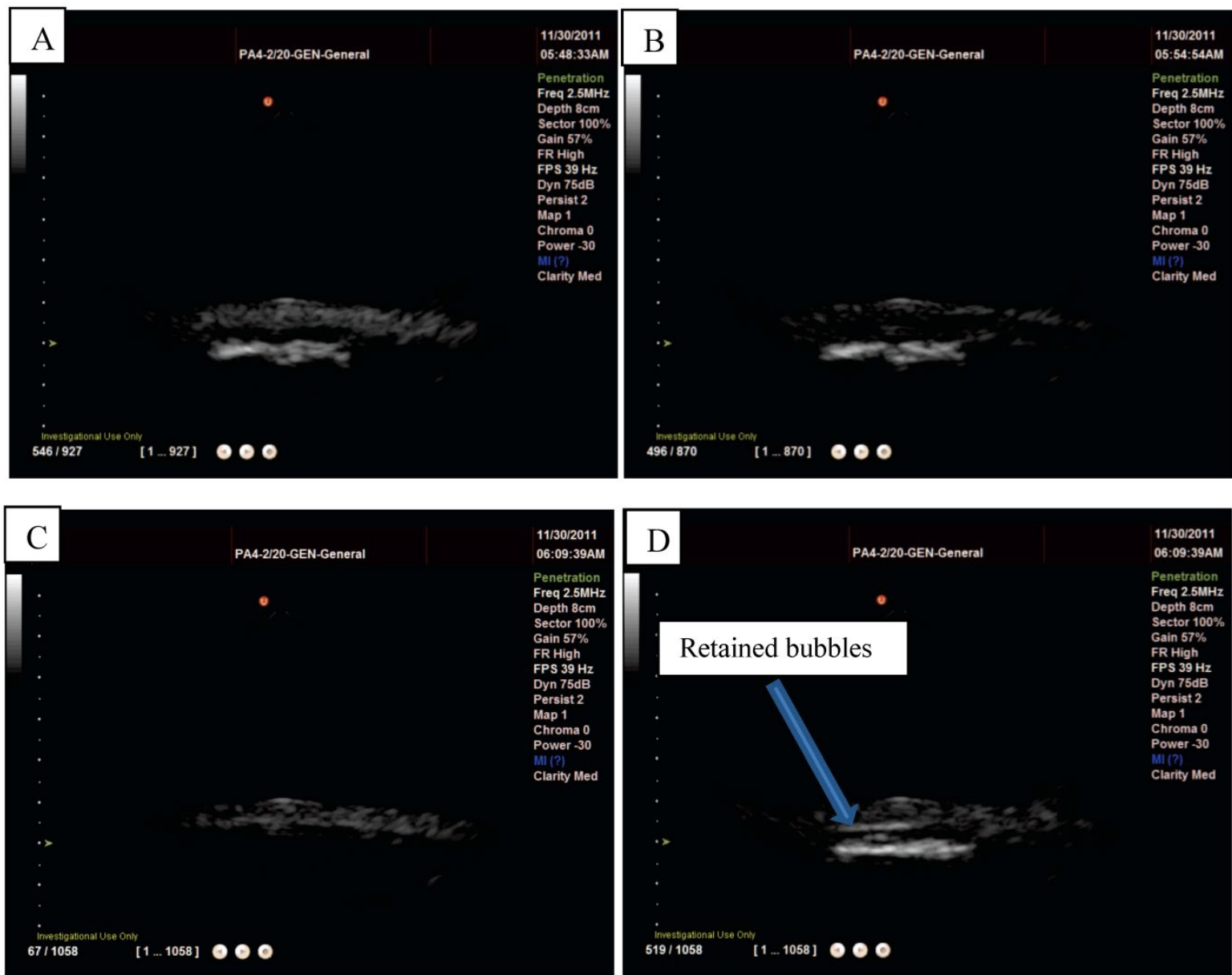

Figure 10: Magnetic droplets and microbubbles being retained under flow in vitro: A) non-magnetic microbubbles showing no retention upon application of a magnetic field (distributed throughout the tube), B) magnetic droplets showing no ultrasound signal when flowing through a latex tube (centre of the tube appears dark), C) a mixture of magnetic droplets and microbubbles flowing through the tube (again tube is filled with echogenic material) and $\mathrm{D}$ ) being retained by a magnetic field.

\section{Discussion}

The optical microscopy images (Figure 4) appear to indicate that there was no interaction between the magnetic droplets and the microbubbles upon mixing. On this basis it might be concluded that the increased levels of transfection observed in the previous gene delivery experiments (19) were due to an interaction between the droplets and the cells, which potentiated the subsequent effect of the microbubble oscillations. The fluorescence images, however, suggest otherwise (Figure 6). Upon mixing with the droplets, the initially non-fluorescent bubbles were seen to fluoresce, indicating that the magnetic droplets were coating or coalescing with the microbubble surface. The fact that the bubbles remained fluorescent after centrifuging suggests that the interaction produced strong binding. To investigate whether the magnetic nanoparticles were integral to the interaction, isoparaffin-only fluorescent droplets were synthesised and the process was repeated. The microbubbles were still observed to fluoresce as shown in Figure 7.

It was evident from its colour, even under optical microscopy, that the magnetic droplet suspension contained objects on the nano- as well as the microscale which cannot be resolved optically. This is supported by results in a recent publication regarding magnetoliposomes which showed that oleic acid coated iron oxide nanoparticles aggregated when mixed with phospholipid in aqueous solution (24). These magnetic droplets most likely interact with the phospholipid microbubbles. Evidence for this is that when the microbubbles fluoresce, microscale vesicles are still fluorescing nearby and optical microscope images appear to show no interaction between the microscale droplets and the microbubbles. As the microbubbles now fluoresce it is reasonable to assume that interaction must come from nanoscale droplets.

The interaction appeared to be strong enough to withstand centrifugation and resist hydrodynamic 
forces within an aqueous suspension. The influence of different species in the suspending medium on the interaction was not investigated but the microbubble and the magnetic droplets are both coated in phospholipids with polar head groups oriented towards the water and as such should be stable. To the authors' knowledge, no studies or models have been reported which examine the interaction between bubbles and droplets. Studies have been performed however on lecithin vesicles where large vesicles grow at the expense of smaller ones due to size disproportionation or Ostwald ripening (25) and one possibility is that it is a similar mechanism by which droplets and microbubbles form magnetic microbubbles. Such a process is determined by energetic stability, small droplets are kinetically stable whereas large droplets are thermodynamically stable; due to the surface to volume ratio (26).

In order to obtain an approximate comparison of the relative surface energies of the microbubble and the magnetic droplet, the Laplace pressure must be calculated using:

$$
P_{\sigma}=\frac{2 \sigma}{R}
$$

Where $\sigma$ is the interfacial tension and $R$ is the radius of the bubble or droplet. The surface energy can then be found as:

$$
F=\sigma \int d A-P_{\sigma} \int d V
$$

Where $A$ is the surface area and $V$ is the volume. The interfacial tension for an air and water interface is $72.75 \mathrm{mN} / \mathrm{m}$ at room temperature, and for water and isoparaffin is $48 \mathrm{mN} / \mathrm{m}$ (27). The microbubbles had a modal diameter, from Figure 4, of $2.5 \mu \mathrm{m}$. The droplets coating the microbubbles must be on the nanoscale and a variable size between 20 and $200 \mathrm{~nm}$ was assumed. With these figures a surface energy of $2.38 \times 10^{-12} \mathrm{Nm}$ is obtained for a $2.5 \mu \mathrm{m}$ air microbubble in water which is lower than $-1.01 \times 10^{-16} \mathrm{Nm}$ for a $20 \mathrm{~nm}$ oil droplet. A $200 \mathrm{~nm}$ droplet has a surface energy of $-1.01 \times 10^{-14} \mathrm{Nm}$ which is still lower than for a microbubble. A $2 \mu \mathrm{m}$ droplet has a surface energy of $-1.01 \times 10^{-12} \mathrm{Nm}$ which is of the same order of magnitude as the microbubble. This indicates that a nanoscale droplet would be likely to interact with the microbubble whereas a micrometre scale droplet would not.

These calculations do not take into account the lipid coating of the droplet or the microbubble. The presence of a lipid monolayer would lower the interfacial tension in both cases. However, the system would still be expected to adopt a more energetically favourable state and lipids are known to mediate fusion between vesicles and membranes (28). The in- fluence of additional membrane components such as polyethylene glycol (PEG), which are present in commercially available microbubbles to aid biocompatibility, might inhibit interaction between microbubbles and droplets but the aim of this study was to investigate the microbubble compositions used in [19] which did not contain PEG.

\section{I. Flow phantom results}

The interaction between the magnetic droplets and the microbubbles to form magnetic microbubbles was further reinforced by the flow phantom studies. Upon optical observation of the magnetic droplet/microbubble mixture in a $200 \mu \mathrm{m}$ capillary tube, objects similar to magnetic microbubbles were observed and responded upon application of a magnetic field as shown in Figure 8. The whole population responded in the absence of flow and a subpopulation was retained against shear rates of approximately 20 $\mathrm{s}^{-1}$. It was, however, difficult to distinguish optically between magnetic microbubbles and large magnetic droplets; but under ultrasound imaging it was shown that particles that were both magnetically and acoustically responsive could be successfully retained by a magnetic field against shear rates of up to $\sim 60 \mathrm{~s}^{-1}(10)$.

These results also indicate that magnetic microbubbles can be retained under physiologically relevant flow conditions. Blood flow rates and wall shear stresses vary considerably throughout the body. For example, the mean shear rate can vary from $40 \mathrm{~s}^{-1}$ in the Venae cavae to $580 \mathrm{~s}^{-1}$ in the capillaries (29) (30). Evidence of successful retention in vivo has already been shown in a mouse model (31) but further work is required to assess the feasibility of retention over a wider range of shear rates and of generating the required magnetic field strengths and gradients at larger tissue depths.

\section{Summary and Conclusions}

The aim of this study was to investigate the mechanisms underlying the results of a previous investigation in which it was shown that a mixture of magnetic liquid droplets and non-magnetic microbubbles was more effective for gene delivery than a suspension of magnetic microbubbles. Two possible mechanisms were proposed: that the magnetic droplets sensitised the cells to sonoporation or that some form of association between the droplets and the microbubbles occurred producing particles that were both magnetically and acoustically responsive.

The observations under optical microscopy in this study were in agreement with those from the previous work and seemed to indicate that no interaction took place between the droplets and particles. 
Optical images of the mixed suspension in a capillary tube in the presence of a magnetic field however indicated particles that were buoyant (and therefore not magnetic liquid droplets) and did respond upon application of a magnetic field. Fluorescence microscopy confirmed that initially non-fluorescent bubbles became coated with fluorescent droplets and that the interaction was sufficiently strong to resist centrifugation. Further examination indicated that the coating was effected by nanoscale liquid droplets and this was why the interaction was not visible under optical microscopy. Surface energy calculations also showed that the magnetic droplets had a lower surface energy than the lipid-coated microbubbles; consequently, adsorption of the droplets to the microbubbles was energetically favourable. Thus it may be reasonably concluded that the results obtained in the previous gene delivery study may be attributed to this interaction. The reason for the higher transfection rates is possibly due to a higher concentration of magnetically and acoustically responsive particles being formed than that present in the magnetic microbubble suspension. Alternatively the response to the ultrasound and/or magnetic field may have been different and this will require further investigation.

Both the magnetic microbubbles and the particles formed from the mixed suspension were echogenic and readily detected under ultrasound imaging. They could also be retained by a magnetic field at centre line velocities of $6 \mathrm{~cm} \mathrm{~s}^{-1}$ and shear rates of $\sim 60$ $\mathrm{s}^{-1}$. These results are promising with respect to the use of magnetic microbubbles as therapeutic delivery vehicles in vivo. These successful preliminary tests need to be fully explored, however. In particular, experiments are required to examine the factors that affect retention: a wider range of flow rates and vessel sizes; the magnitude as well as the gradient of the magnetic field; the distance between the magnet and the vessel (i.e. tissue depth); the liquid density, viscosity and pulsatility. Further investigation of the particle internal structure and their performance in therapeutic delivery will also be required.

\section{Acknowledgements}

The authors would like to thank The Leverhulme Trust, The Engineering and Physical Sciences Research Council, University College London and The Royal Institution of Great Britain for contributing funding towards this research. They would also like to thank Ms. Graciela Mohamedi, Dr. Neveen Hosny, Dr. Julian George and Prof. Constantin Coussios for their assistance and advice.

\section{Competing Interests}

The authors have declared that no competing interest exists.

\section{References}

1. Pankhurst QA, Connolly J, Jones SK, Dobson J. Applications of magnetic nanoparticles in biomedicine. Journal of Physics D: Applied Physics 2003;36(13):R167-R81.

2. Ferrara K, Pollard R, Borden M. Ultrasound microbubble contrast agents: Fundamentals and application to gene and drug delivery. Annual Review of Biomedical Engineering 2007;9:415-47.

3. Unger EC, Hersh E, Vannan M, Matsunaga TO, McCreery T. Local drug and gene delivery through microbubbles. Progress in Cardiovascular Diseases 2001;44(1):45-54.

4. Harvey CJ, Pilcher JM, Eckersley RJ, Blomley MJK, Cosgrove DO. Advances in ultrasound. Clinical Radiology 2002;57(3):157-77.

5. Schlicher RK, Radhakrishna H, Tolentino TP, Apkarian RP, Zarnitsyn V, Prausnitz MR. Mechanism of intracellular delivery by acoustic cavitation. Ultrasound Med Biol 2006;32(6):915-24.

6. Rahim A, Taylor SL, Bush NL, ter Haar GR, Bamber JC, Porter CD. Physical parameters affecting ultrasound/microbubble-mediated gene delivery efficiency in vitro. Ultrasound Med Biol 2006;32(8):1269-79.

7. Thanh NTK. Magnetic Nanoparticles: From Fabrication to Clinical Applications. Taylor and Francis; 2011.

8. Zimmerman U, Scheurich P, Pilwat G, Benz R. Cells with manipulated functions: New perspectives for cell biology, medicine, and technology. Angewandte Chemie - International Edition in English 1981;20(4):325-44.

9. Senyei AE, Reich SD, Gonczy C, Widder KJ. In vivo kinetics of magnetically targeted low-dose doxorubicin. Journal of Pharmaceutical Sciences 1981;70(4):389-91.

10. Widder KJ, Senyei AE, Ranney DF. In vitro release of biologically active adriamycin by magnetically responsive albumin microspheres. Cancer Research 1980;40(10):3512-7.

11. Scherer F, Anton M, Schillinger U, Henke J, Bergemann C, Krüger A, Gänsbacher B, Plank C. Magnetofection: Enhancing and targeting gene delivery by magnetic force in vitro and in vivo. Gene Therapy 2002;9(2):102-9.

12. Plank C, Zelphati O, Mykhaylyk O. Magnetically enhanced nucleic acid delivery. Ten years of magnetofection-Progress and prospects. Advanced Drug Delivery Reviews 2011;63(14-15):1300-31.

13. Soetanto K, Watarai H. Development of magnetic microbubbles for drug delivery system (DDS). Japanese Journal of Applied Physics, Part 1: Regular Papers and Short Notes and Review Papers 2000;39(5 B):3230-2.

14. Soetanto K, Watarai H. Ferromagnetic Ultrasound Microbubbles Contrast Agent. Cancun. 2003.

15. Yang F, Li L, Li Y, Chen Z, Wu J, Gu N. Superparamagnetic nanoparticle-inclusion microbubbles for ultrasound contrast agents. Physics in Medicine and Biology 2008;53(21):6129-41.

16. Yang F, Li Y, Chen Z, Zhang Y, Wu J, Gu N. Superparamagnetic iron oxide nanoparticle-embedded encapsulated microbubbles as dual contrast agents of magnetic resonance and ultrasound imaging. Biomaterials 2009;30(23-24):3882-90.

17. Vlaskou D, Mykhaylyk O, Krötz F, Hellwig N, Renner R, Schillinger U, Gleich B, Heidsieck A, Schmitz G, Hensel K, Plank C. Magnetic and Acoustically Active Lipospheres for Magnetically Targeted Nucleic Acid Delivery. Advanced Functional Materials 2010;20(22):3881-94.

18. Vlaskou D, Pradhan P, Bergemann C, Klibanov AL, Hensel K, Schmitz G, Plank C, Mykhaylyk O. Magnetic microbubbles: Magnetically targeted and ultrasound-triggered vectors for gene delivery in vitro. Rostock. 2010 .

19. Stride E, Porter C, Prieto AG, Pankhurst Q. Enhancement of Microbubble Mediated Gene Delivery by Simultaneous Exposure to Ultrasonic and Magnetic Fields. Ultrasound in Medicine \& Biology 2009;35(5):861-8.

20. Kuimova MK, Yahioglu G, Levitt JA, Suhling K. Molecular rotor measures viscosity of live cells via fluorescence lifetime imaging. Journal of the American Chemical Society 2008;130(21):6672-3.

21. Sennoga CA, Mahue V, Loughran J, Casey J, Seddon JM, Tang M, Eckersley RJ. On sizing and counting of microbubbles using optical microscopy. Ultrasound Med Biol 2010;36(12):2093-6.

22. Willert CE, Gharib M. Digital particle image velocimetry. Experiments in Fluids 1991;10(4):181-93.

23. Verkaik AC, Beulen BWAMM, Bogaerds ACB, Rutten MCM, van de Vosse FN. Estimation of volume flow in curved tubes based on analytical 
and computational analysis of axial velocity profiles. Physics of Fluids 2009;21(2).

24. Amstad E, Kohlbrecher J, Müller E, Schweizer T, Textor M, Reimhult E. Triggered release from liposomes through magnetic actuation of iron oxide nanoparticle containing membranes. Nano Letters 2011;11(4):1664-70.

25. Johnson NW, Kaler EW. Size disproportionation in vesicular dispersions. Journal of Colloid and Interface Science 1987;116(2):444-57.

26. Taylor P. Ostwald ripening in emulsions. Advances in Colloid and Interface Science 1998;75(2):107-63.

27. [Internet] NPL. http://www.kayelaby.npl.co.uk/general_physics/ 2_2/2_2_5.html

28. Mouritsen O. Life - As a Matter of Fat. Springer; 2001.

29. Whitmore RL. The flow behaviour of blood in the circulation. Nature 1967;215(5097):123-6.

30. Eckmann DM, Bowers S, Stecker M, Cheung AT. Hematocrit, volume expander, temperature, and shear rate effects on blood viscosity. Anesthesia and Analgesia 2000;91(3):539-45.

31. Mulvana H, Eckersley RJ, Browning R, Hajnal JV, Stride E, Barrack T, Tang M, Pankhurst Q, Wells D. Enhanced gene transfection in vivo using magnetic localisation ofultrasound contrast agents: Preliminary results. San Diego, CA. 2010 